\title{
Engineering of optical and electronic band gaps in transition metal dichalcogenide monolayers through external dielectric screening
}

\author{
Sven Borghardt, ${ }^{1,{ }^{*}}$ Jhih-Sian Tu, ${ }^{1}$ Florian Winkler, ${ }^{2,3}$ Jürgen Schubert, ${ }^{1}$ Willi Zander, ${ }^{1}$ \\ Kristjan Leosson, ${ }^{4,5}$ and Beata E. Kardynał ${ }^{1, \dagger}$ \\ ${ }^{1}$ Peter Grünberg Institute 9 (PGI-9), Forschungszentrum Jülich, DE-52425 Jülich, Germany \\ ${ }^{2}$ Ernst Ruska-Centre for Microscopy and Spectroscopy with Electrons (ER-C), Forschungszentrum Jülich, DE-52425 Jülich, Germany \\ ${ }^{3}$ Peter Grünberg Institute 5 (PGI-5), Forschungszentrum Jülich, DE-52425 Jülich, Germany \\ ${ }^{4}$ Innovation Center Iceland, Keldnaholti, IS-112 Reykjavik, Iceland \\ ${ }^{5}$ Science Institute, University of Iceland, Dunhagi 5, IS-107 Reykjavik, Iceland
}

(Received 18 July 2017; revised manuscript received 10 August 2017; published 4 October 2017)

\begin{abstract}
Heterojunctions are the backbone of established semiconductor technology. The highly desirable reliable creation of dielectrically defined heterojunctions in transition metal dichalcogenide monolayers (TMD-MLs) requires an in-depth understanding of dielectric screening effects induced by the ML's environment. Here we report on the modulations of excitonic transitions in TMD-MLs through the effect of dielectric environments including low- $k$ and high- $k$ dielectric materials. We present absolute tuning ranges as large as $37 \mathrm{meV}$ for the optical band gaps of $\mathrm{WSe}_{2}$ and $\mathrm{MoSe}_{2}$ MLs and relative tuning ranges on the order of $15 \%$ for the binding energies of charged excitons. Additionally, we measure relative changes of 30\% in the energy splittings of exciton Rydberg states of $\mathrm{WSe}_{2}$. The findings enable us to estimate changes in the exciton binding energies and the electronic band gaps of the studied materials.
\end{abstract}

DOI: 10.1103/PhysRevMaterials.1.054001

\section{INTRODUCTION}

Since the discovery of graphene the class of atomically thin two-dimensional materials has been growing continuously [1-4]. An exciting expansion of this class was done when transition metal dichalcogenide monolayers (TMD-MLs) were added to this material class [5]. In contrast to the semimetal graphene, TMD-MLs with the stoichiometric formula $\mathrm{MX}_{2}$, where $\mathrm{M}$ and $\mathrm{X}$ denote a transition metal atom $(\mathrm{Mo}, \mathrm{W}, \ldots)$ and a chalcogen atom $(\mathrm{S}, \mathrm{Se}, \ldots)$, respectively, are semiconductors with direct band gaps at the $K$ points of the hexagonal Brillouin zone with energies in the range of visible light [6]. Additionally, the heavy metal ions, the reduced Coulomb screening in two dimensions, and the lack of inversion symmetry in these materials result in a unique combination of giant exciton binding energies, large spin-orbit coupling, as well as a coupling between the spin and valley degrees of freedom at the two inequivalent $K$ valleys that can be accessed optically [7-9].

Although there have been numerous reports on electronic and optoelectronic nanodevices made from TMD-MLs, such as single-layer transistors, light-emitting diodes, and photodetectors [10-12], there is a strong need to develop methods to create junctions. In analogy to well established semiconductor technology, where heterojunctions are the building blocks of most electronic and optoelectronic devices (e.g., laser diodes, HEMT transistors), the reliable creation of lateral heterojunctions within TMD-MLs would enable further development of next generation optoelectronic devices.

Similarly to three-dimensional semiconductors where heterostructures are formed by interfacing materials of different chemical composition, lateral heterostructures of TMDs have been developed by introducing laterally varying stoichiometry

\footnotetext{
*s.borghardt@fz-juelich.de

†b.kardynal@fz-juelich.de
}

$[13,14]$. An alternative approach, which is unique to low dimensional materials, relies on the local modulation of screening of the ionic Coulomb potentials, as well as the Coulomb interaction between charge carriers, within the ML through a local variation of the relative permittivity of the ML's direct environment, i.e., its substrate and cover material $[15,16]$.

Figure 1 depicts how externally induced screening changes both the electronic band gap $E_{g}$ and the binding energies of exciton complexes in a TMD-ML. The binding energy for the neutral exciton $E_{b, x}$ is given by the difference between $E_{g}$ and the ground state energy of the neutral exciton $E_{x, 1}$ :

$$
E_{b, x}=E_{g}-E_{x, 1} .
$$

In addition to the neutral exciton, charged excitons (trions), which show a negative polarity in unbiased devices, have been observed in TMD-MLs [17-20]. The binding energy of the trion $E_{b, x^{-}}$is given by the energy difference between $E_{x, 1}$ and the trion energy $E_{x^{-}}$:

$$
E_{b, x^{-}}=E_{x, 1}-E_{x^{-}} .
$$

TMD-MLs show neutral exciton binding energies of several hundreds of meV [7,21-23] and trion binding energies on the order of $30 \mathrm{meV}$ [17-20]. Both the electronic band gap $E_{g}$ and the binding energies of exciton complexes $E_{b, x}$ and $E_{b, x^{-}}$are expected to decrease with an increasing relative permittivity of the surrounding dielectric $[15,16,24,25]$. According to Eq. (1) the sign of the change of the optical band gap, which is equivalent to $E_{x, 1}$, depends on the relative change of $E_{g}$ and $E_{b, x}$.

A first experimental evidence for the tunability of excitonic emission energies in TMD-MLs through environment-induced dielectric screening has been the report of a room temperature blue shift of $E_{x, 1}$ and $E_{x^{-}}$in $\mathrm{MoS}_{2}$ MLs with an increasing relative permittivity of the nonionic liquid with which the samples were covered [26]. In addition to changes in the 

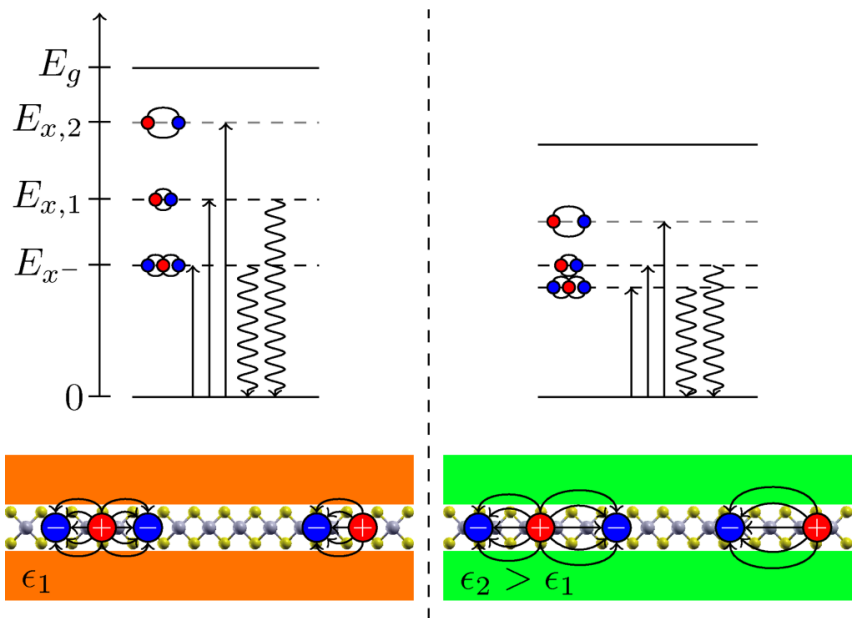

FIG. 1. Schematic diagram of externally induced dielectric screening effects on the electronic band gap and the excitonic states in TMD-MLs. Solid and waved arrows represent optical absorption and radiative recombination processes, respectively. The $k$ dispersion of excitons is not shown here.

emission energies of excitons, an increase of the exciton Bohr radius in $\mathrm{WSe}_{2}$ MLs with an increasing background relative permittivity of the surrounding material of the ML has been observed [27]. Furthermore, a decrease of the exciton binding energy and the electronic band gap has been observed for $\mathrm{WS}_{2}$ MLs stacked with graphene [28]. More recently, Lippert et al. have reported on a lack of a trend in excitonic emission energies as a function of the refractive index of the substrate [29]. However, the latter report omits a discussion of important aspects, such as the chemical properties of the different substrate surfaces or the well-known spatial variation of exciton emission energies.

In this work we investigate different types of dielectrics as potential components for lateral heterostructures. We employ low temperature $\mu$-photoluminescence, as well as reflectance measurements, to study $\mathrm{MoSe}_{2}$ and $\mathrm{WSe}_{2} \mathrm{MLs}$. Our results show a redshift of the optical band gaps, as well as a reduction of the binding energies of both the neutral exciton and the trion, with an increasing relative permittivity of the ML's surrounding. Estimates on the resulting changes of the electronic band gaps are made from the measured optical band gaps and exciton binding energies.

\section{EXPERIMENTS}

Mechanically exfoliated $\mathrm{WSe}_{2}$ and $\mathrm{MoSe}_{2}$ MLs have been prepared on different substrates (see the Supplemental Material for more information [30]). The substrates were required to be flat in order to minimize the strain and the number of defects introduced in the TMD-MLs. Only transparent wide band gap materials were used. The substrates considered in this work can be separated into three types [Figs. 2(a)-2(c)] according to their surface properties. The first type of substrate, namely CYTOP, is a low- $k$ fluoropolymer, which is known for its chemical inertness and hydrophobicity. It is amorphous and transparent across a wide wavelength range and it features a very low relative permittivity in the entire frequency range. The second type are stable, transparent (a)

(c)

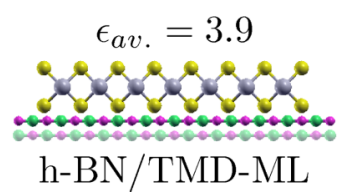

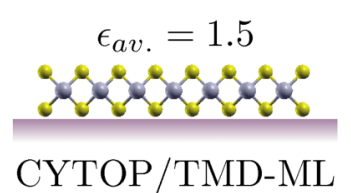

(b)

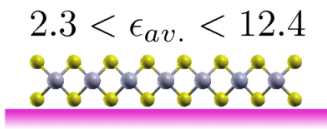

Oxide/TMD-ML

(d)

$\epsilon_{a v}=6.9$

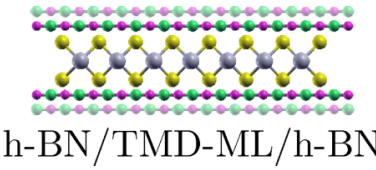

FIG. 2. Symbolic diagram of the structures studied in this work, together with the average relative permittivity of the substrate and cover material in each structure.

oxide substrates. Oxides offer a wide range of $k$ values but they are known to be hydrophilic. Hexagonal boron-nitride (h-BN) is a wide band gap layered material that can form van der Waals heterostructures with TMD-MLs, which have been used to improve the optical properties of TMD-MLs [31,32], as well as to study new physical phenomena in two-dimensional materials, such as exciton-phonon coupling [33,34]. Similarly to CYTOP, h-BN is hydrophobic. A TMD-ML encapsulated by two hexagonal h-BN flakes was also included as a fourth dielectric environment [Fig. 2(d)].

On a theoretical level, the effect of dielectric screening on excitonic properties is complex and nonanalytic. Especially, an open question remains in which frequency domains the screening occurs. In our analysis, for the sake of simplicity, we use the static dielectric constant of materials. This represents a simplification, but, as elaborated in the Supplemental Material [30], does not change the conclusions from this work. The average nominal relative permittivity of the environment $\epsilon_{\mathrm{av} .}=$ $\frac{1}{2}\left(\epsilon_{\text {top }}+\epsilon_{\text {bottom }}\right)$ ranged from 1.5 for a TMD-ML on CYTOP to 12.4 for a TMD-ML on $\mathrm{LaAlO}_{3}$. All the layers in contact with the MLs were at least several nanometers thick and, as a consequence, only the layers in direct contact with the TMDML are needed to be included in the analysis.

$\mu$-photoluminescence maps, as well as optical reflectance measurements of all structures were acquired at $11 \mathrm{~K}$. While the former method only probes the emission energies of the ground states of neutral excitons and trions, absorption energies of the ground state, as well as of excited states, of the neutral exciton can be probed by the latter [21,23]. We further analyze the impact of charging induced by the substrate or the cover material as the reason for the observed effects by performing gate-dependent experiments.

\section{RESULTS AND DISCUSSIONS}

The emission energies of the neutral exciton and the trion of all structures shown in Fig. 2 have been measured in $\mu$-photoluminescence experiments. Figure 3 shows photoluminescence spectra for three representative structures. The spectra contain a signal from neutral exciton and trion recombination, as well as defect emission in the case of $\mathrm{WSe}_{2}$ MLs. The energy of these peaks shows a systematic shift with 


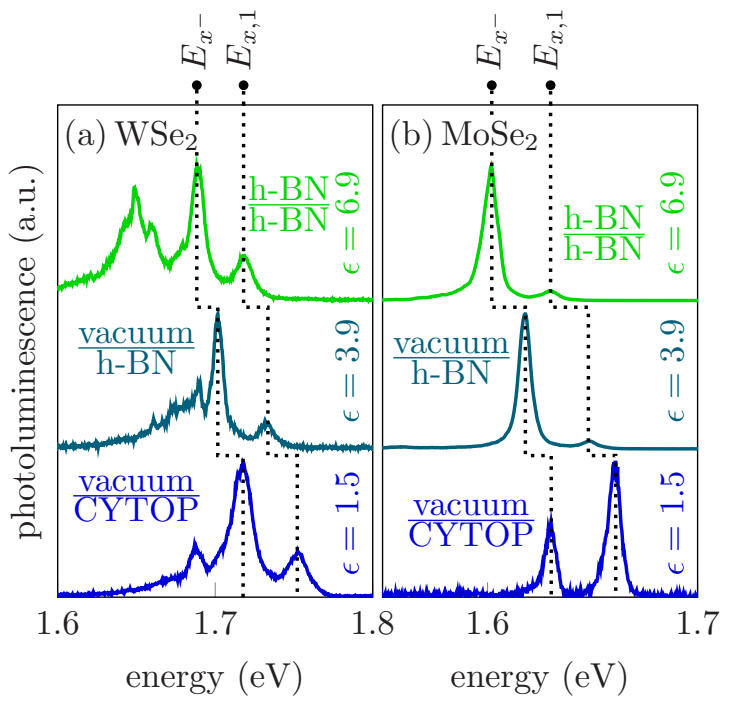

FIG. 3. Representative photoluminescence spectra for $\mathrm{WSe}_{2}$ (a) and $\mathrm{MoSe}_{2}$ (b) MLs in different dielectric environments. The highest and second highest energy peak in each spectrum represent the neutral exciton and trion emission, respectively. For $\mathrm{WSe}_{2}$, a band of defects can be observed at the low energy end of the spectra. The dotted black lines connect the neutral exciton and trion emission peaks of each spectrum, as a guide to the eye.

$\epsilon_{\text {av. }}$. Figure 4 summarizes the emission energies for both the neutral exciton and the trion for all measured structures. The data points are averaged over large areas in order to account for spatial variations of the peak positions. The trends in behavior of $\mathrm{WSe}_{2}$ and $\mathrm{MoSe}_{2} \mathrm{MLs}$ in different environments are very similar. The results for both TMD-MLs on $\mathrm{SiO}_{2}$, which is the most commonly used substrate, agree well with reports in the literature $[17,35]$. There is a clear correlation in the positions of neutral exciton and trion peaks but there is no clear correlation with the nominal $\epsilon_{\mathrm{av}}$. Irrespective of the nominal relative permittivity of the substrate, the emission energies for MLs on CYTOP and for MLs on oxide substrates are very similar to each other and cluster around $\left(E_{x, 1}, E_{x^{-}}\right)=(1.754 \mathrm{eV}, 1.717 \mathrm{eV})$ for $\mathrm{WSe}_{2}$ and at $\left(E_{x, 1}, E_{x^{-}}\right)=(1.664 \mathrm{eV}, 1.631 \mathrm{eV})$ for $\mathrm{MoSe}_{2}$. We attribute this lack of dielectric effects to the presence of water on the hydrophilic oxide surface preventing a direct contact between the substrate material and the TMD-MLs. Hexagonal ice has a relative permittivity below 2 and, thus, similar to that of CYTOP [36]. In the case of $\mathrm{LaAlO}_{3}$ as a substrate, a blueshift of the exciton emission energies with respect to other oxide substrates can be observed especially for $\mathrm{MoSe}_{2}$. So far we are not able to fully explain this behavior, which is in contrast to the h-BN structures. A possible explanation may be a different range of dielectric effects acting on the electronic band gap and the excitonic binding energies. In this scenario, the effects on the former quantity could be quenched by a thin layer of ice, whereas the the latter quantity still experiences the presence of the very high relative permittivity of $\mathrm{LaAlO}_{3}$. Proving this theory, however, requires further investigations.

Even though the relative permittivity of h-BN is lower than that of some oxides (see the Supplemental Material [30]), the emission energies of MLs on a h-BN substrate, as well as of MLs encapsulated in h-BN, show clearly observable redshifts with respect to this cluster. For the neutral exciton, the redshifts with respect to a ML on CYTOP are $17 \mathrm{meV}$ (16 meV) and $35 \mathrm{meV}(34 \mathrm{meV})$ for $\mathrm{WSe}_{2}\left(\mathrm{MoSe}_{2}\right) \mathrm{MLs}$ on h-BN and encapsulated with h-BN, respectively. For the trion, the redshifts with respect to a ML on CYTOP are

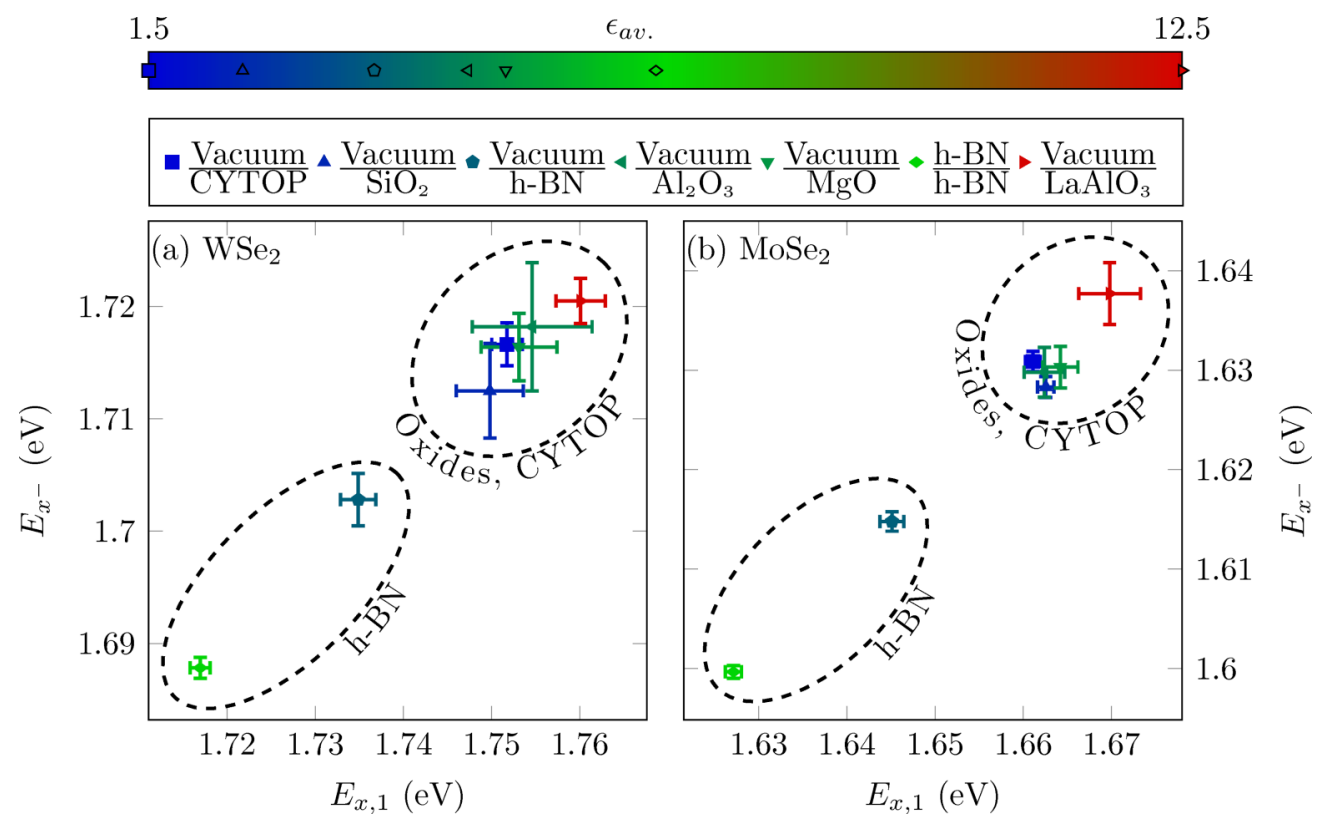

FIG. 4. Emission energies of the neutral exciton and the trion in $\mathrm{WSe}_{2}$ (a) and $\mathrm{MoSe}_{2}$ (b) MLs for different dielectric environments. The legend shows the substrate and the cover material below and above the horizontal line, respectively. The color of the plotted data represents $\epsilon_{\mathrm{av}}$. The emission energies at each sample position have been obtained by hyperspectral fitting using a purpose-developed software (see the Supplemental Material [30]) and the mean values and error bars are obtained by a pixel-by-pixel averaging over the sample area. 


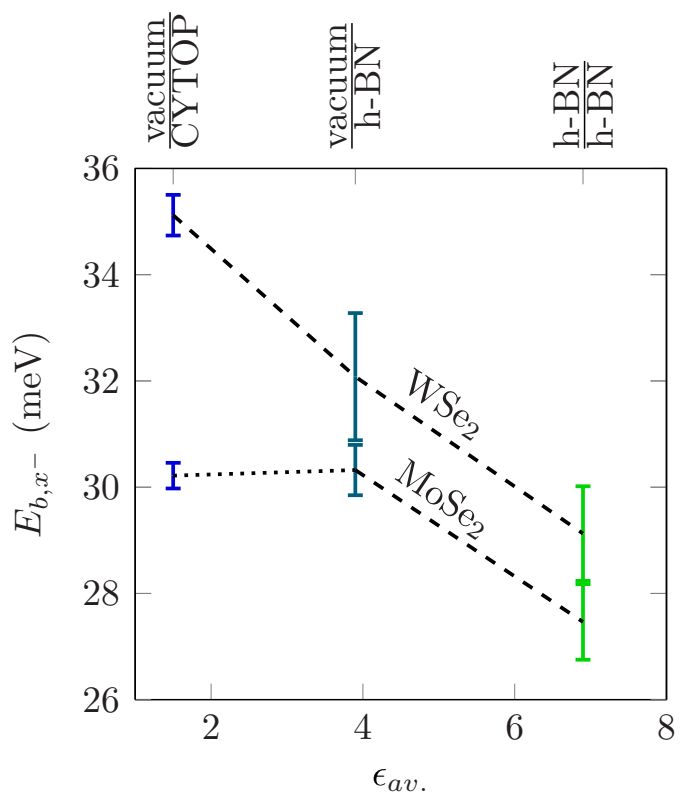

FIG. 5. Trion binding energies in $\mathrm{WSe}_{2}$ and $\mathrm{MoSe}_{2}$ MLs as a function of $\epsilon_{\mathrm{av}}$. The binding energies at each sample position have been obtained by hyperspectral fitting using a purpose-developed software (see the Supplemental Material [30]) and the mean values and error bars are obtained by a pixel-by-pixel averaging over the sample area.

$14 \mathrm{meV}(16 \mathrm{meV})$ and $29 \mathrm{meV}(31 \mathrm{meV})$ for $\mathrm{WSe}_{2}\left(\mathrm{MoSe}_{2}\right)$ MLs on h-BN and encapsulated with h-BN, respectively. In contrast to the interface between oxides and TMD-MLs, it is well known that contaminations are highly mobile in mechanically stacked van der Waals heterostructures clustering into so-called bubbles leaving behind large areas of clean interfaces (Supplemental Material, Fig. 1 [30]) [37]. Since this contamination covers only a very small fraction of the sample area, the photoluminescence emission from the clean interfaces is expected to dominate the measured signal.

The observed redshifts for the h-BN structures imply that the electronic band gap is undergoing a larger absolute change than the exciton binding energies when increasing $\epsilon_{\mathrm{av}}$. [Eq. (1)]. These findings are in contrast to previously reported observations in room temperature measurements of $\mathrm{MoS}_{2} \mathrm{MLs}$ in dielectric nonionic liquids [26], but agree well with recent reflectance studies of TMD-MLs stacked with graphene [28].

Figure 5 shows the evolution of the trion binding energy, determined from the difference between neutral exciton and trion energies [Eq. (2)], as a function of $\epsilon_{a v}$, for $\mathrm{MoSe}_{2}$ and $\mathrm{WSe}_{2}$. For $\mathrm{WSe}_{2}$ the trion binding energy decreases monotonically from $35 \mathrm{meV}$ for a ML on CYTOP to $29 \mathrm{meV}$ for a ML encapsulated in h-BN. This result is in good qualitative agreement with theoretical predictions [25]. Similarly to $\mathrm{WSe}_{2}$, the trion binding energy of $\mathrm{MoSe}_{2}$ decreases from $30 \mathrm{meV}$ for a ML on h-BN to $27 \mathrm{meV}$ for a ML encapsulated between two h-BN flakes. However, in contrast to $\mathrm{WSe}_{2}$, the trion binding energy of a $\mathrm{MoSe}_{2} \mathrm{ML}$ is nearly identical for a ML on CYTOP and a ML on h-BN. This small change is still under investigation. A possible explanation may be a positive polarity of the trion for a ML on CYTOP, which, having a lower binding energy than negatively charged trions, would cancel out the effect of the reduced screening [35]. An indication for a different background charge for the ML on CYTOP is the different exciton-trion ratio observed in this structure (Fig. 3). The trion binding energies are determined by averaging the trion binding energies from all pixels of a $\mu$-photoluminescence map. The standard deviations shown in Fig. 5 are up to five times lower than those determined for the exciton and trion emission energies themselves (Fig. 4). This lower spatial fluctuation of the trion binding energy, confirms the robustness of excitonic binding energies against perturbations such as strain, as expected from theoretical studies [38]. Trion binding energies could thus be potentially used as a sensitive, noninvasive probe of surface state of TMD-MLs, providing an alternative to the use of water droplets [39].

Changes of the optical band gap of TMD-MLs with spatially modulated $\epsilon_{\text {av. }}$ are shown in Fig. 6 for a single $\mathrm{MoSe}_{2}$ ML. The sample is prepared by placing the ML on an interface between $\mathrm{SiO}_{2}$ and h-BN and partially covering it with a h-BN layer. While small variations of $E_{x, 1}$ within a region of constant $\epsilon_{\mathrm{av}}$. are present, the correlation of $E_{x, 1}$ with the local $\epsilon_{\mathrm{av}}$. of the TMD-ML is clear. The residual variations within each region are most likely due to strain variations, which are known to change the ML's electronic band gap [40], as well as its optical band gap [41,42]. A line profile across the boundary that separates the part of the ML encapsulated in h-BN from the uncovered part on h-BN is shown in Fig. 6(c). In the line profile, it can be seen that the change of the optical band gap is observed over a distance of approximately $1.5 \mu \mathrm{m}$, which is the size of the laser spot in our measurements. Consequently, the band gap change is much more abrupt than the radius of the laser spot, which agrees well with theoretical predictions of the transition occurring across only a few crystal unit cells [15]. A representative spectrum from the interface between the two areas is shown in Fig. 6(d). It is a clear superposition of the peaks characteristics of each of the two areas. A line profile crossing the boundary between the ML area on h-BN and the ML area on $\mathrm{SiO}_{2}$ cannot be analyzed, as there is no smooth transition of the photoluminescence signal observable. The height step between the two areas, which introduces strain or defects in the ML, is most likely responsible for the distortions. The comparison between the two discussed interfaces demonstrates that the formation of well-defined, abrupt lateral heterostructures requires a change of the relative permittivity on a flat substrate.

So far the discussion has been limited to photoluminescence experiments, the results of which are of importance mainly for the design of new ultrathin light sources with a tailored emission energy. However, the electronic band gap is also affected by the dielectric screening and an understanding of the interplay between the two values may be of importance for trion confinement, as well as confinement in electronic devices. For this reason, we performed optical reflectance measurements which probe Rydberg series of the neutral exciton. The energy splittings in the Rydberg series are related to the exciton binding energy, the latter combined with the optical band gap yielding an estimate of the electronic band gap [21]. The reflectance measurements were limited to $\mathrm{WSe}_{2}$ MLs. The excited states of the neutral exciton overlap with the $\mathrm{B}$ exciton in $\mathrm{MoSe}_{2}$ MLs and, thus, cannot be measured [44]. 


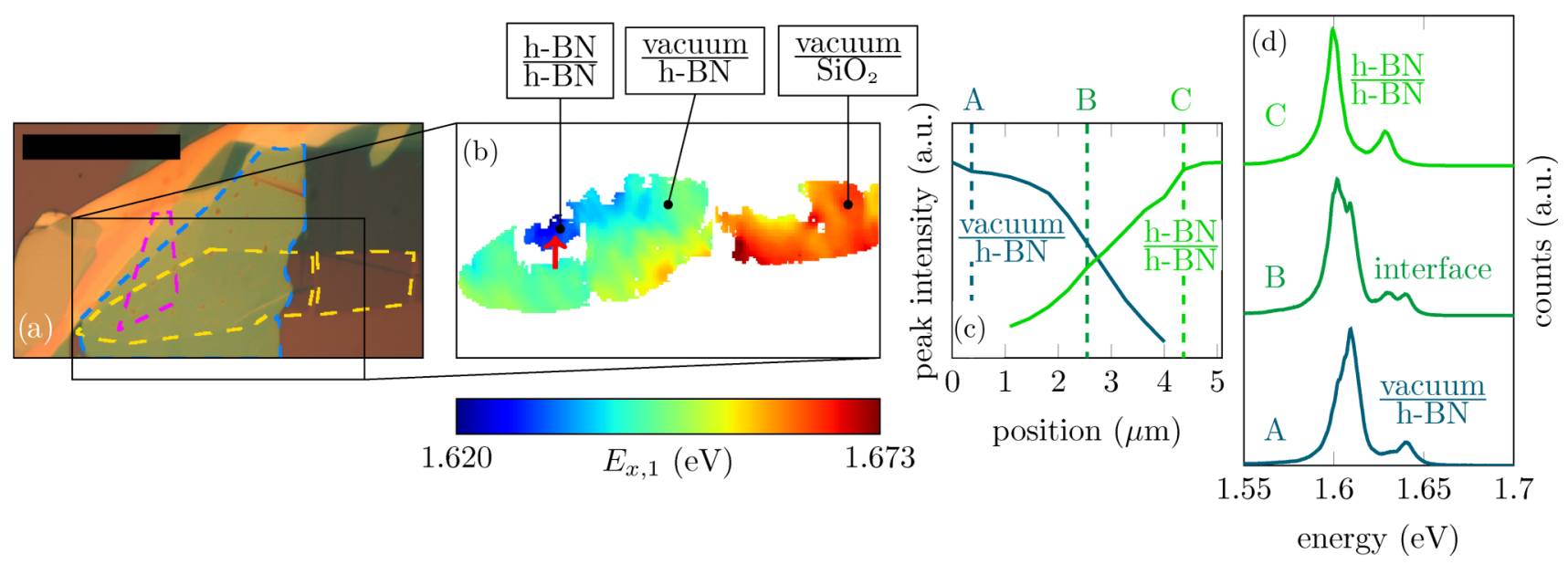

FIG. 6. $\mathrm{MoSe}_{2}$ monolayer in different dielectric environments. (a) Optical micrograph of the TMD-ML (yellow line), which is partly lying on $\mathrm{SiO}_{2}$ substrate and partly lying on a h-BN flake (blue line). Furthermore, a part of the latter sample area is covered with another h-BN flake (magenta line). The scale bar is $20 \mu \mathrm{m}$. (b) Spatial distribution of the exciton emission energy of the TMD-ML. Note that some pixels needed to be removed from the analysis when they showed disturbed photoluminescence spectra or when they probed two dielectric environments at the same time. (c) Normalized intensities for the neutral exciton emission from the ML on h-BN and the ML encapsulated by h-BN along the a path marked by the red arrow in (b). (d) Representative spectra along the same path.

Due to the optical transparency of TMD-MLs, the effect of light reflection from the ML constitutes as a small part of the total reflections from the sample. For this reason, reflectance contrast measurements need to be performed on such structures. The reflectance contrast is then given by $\frac{\Delta R}{R}=\frac{R_{\mathrm{ML}}-R_{\mathrm{ref}}}{R_{\mathrm{rt}}}$, where $R_{\mathrm{ML}}$ and $R_{\text {ref. }}$ denote the reflectance from the ML region and the reflectance from a reference region without a ML structure, respectively. In order to make the features of the excited exciton states more pronounced, it is convenient to plot the energy derivative of the reflectance contrast $\frac{d}{d E} \frac{\Delta R}{R}$ [21].

Figure 7 shows the energy derivative of the reflectance contrast of $\mathrm{WSe}_{2}$ MLs for three measured samples together with the evolution of the energy splitting between the ground state and the first excited state of the neutral exciton $\Delta E_{12}=$ $E_{x, 2}-E_{x, 1}$ as a function of $\epsilon_{\mathrm{av} .}$. As in the photoluminescence experiments, the optical band gap is redshifted. Strikingly, an even stronger redshift is observed for $E_{x, 2}$, as expected theoretically due to the larger separation of the electron and the hole for excited states [45]. As a consequence, it is apparent that $\Delta E_{12}$ is decreasing with an increasing relative permittivity of the surrounding material of the ML. $\Delta E_{12}$ decreases monotonically from approximately $180 \mathrm{meV}$ for a $\mathrm{WSe}_{2} \mathrm{ML}$ on CYTOP to almost $130 \mathrm{meV}$ for a $\mathrm{WSe}_{2} \mathrm{ML}$ encapsulated by two h-BN flakes. The energy splitting for a $\mathrm{WSe}_{2}$ on CYTOP is slightly higher than for $\mathrm{WSe}_{2}$ MLs on $\mathrm{SiO}_{2}$, which has been measured using different techniques and at different temperatures $[22,46]$.

It has been shown that the ratio $\frac{E_{b, x}}{\Delta E_{12}}$ for TMD-MLs strongly deviates from a classic hydrogenic model in two dimensions with $\frac{E_{b, x}}{\Delta E_{12}}=\frac{9}{8}$ [21-23]. For a WSe 2 ML on $\mathrm{SiO}_{2}$ linear absorption spectroscopy combined with twophoton photoluminescence excitation spectroscopy yielded $\frac{E_{b, x}}{\Delta E_{12}}=2.3$ [22]. The exact behavior of $\frac{E_{b, x}}{\Delta E_{12}}$ as a function of the dielectric environment has not been addressed experimentally so far. However, theoretical studies based on the quantum-electrostatic heterostructure model suggest a decrease of this factor if the relative permittivity of the environment increases [47]. Under the assumption of a decreasing ratio of $\frac{E_{b, x}}{\Delta E_{12}}$ with an increasing relative permittivity and using the fact that $\Delta E_{12}$ for a $\mathrm{WSe}_{2} \mathrm{ML}$ on CYTOP is slightly

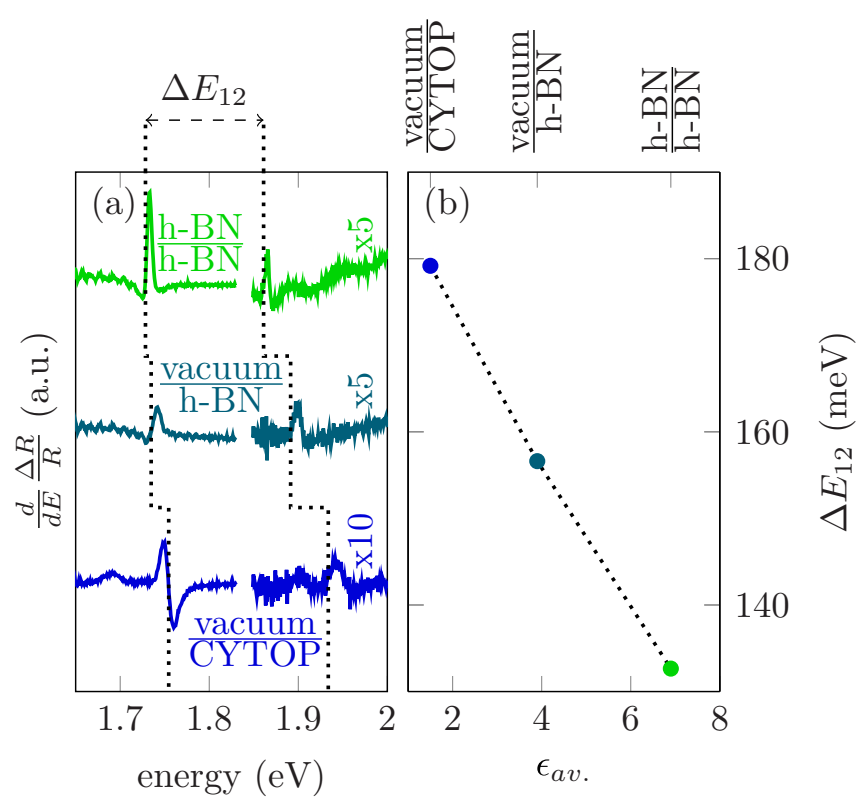

FIG. 7. Reflectance contrast measurements for $\mathrm{WSe}_{2}$ MLs in environments with different relative permittivity. (a) The energy derivative of the reflectance contrast. The dotted lines connect the ground state and the first excited state of the neutral exciton of each spectrum, as a guide to the eye. The positions of the ground state and excited state were determined by optimizing the energy derivative of the reflection contrast calculated using the transfer matrix method [43]. (b) Energy splitting of the ground state and the first excited state of the neutral exciton, as a function of $\epsilon_{\mathrm{av}}$. 
larger than for a $\mathrm{WSe}_{2} \mathrm{ML}$ on $\mathrm{SiO}_{2}$, we can, thus, obtain upper and lower bounds of the changes of the exciton binding energy and the electronic band gap, respectively. We obtain a maximum reduction in the exciton binding energy on the order of $115 \mathrm{meV}$ when replacing the CYTOP substrate with h-BN encapsulation. Combining this result with the measured redshifts in the optical band gap results in a minimum change of the electronic band gap on the order of $150 \mathrm{meV}$, which exceeds the thermal energy at room temperature by a factor of 6 . Further experimental studies, such as scanning tunneling spectroscopy [7] or angle-resolved photoemission spectroscopy [48], are required to obtain an accurate picture of the evolution of the exciton binding energy as a function of the dielectric environment of a TMD-ML.

Besides the dielectric effects, it is well known that emission energies in TMD-MLs are dependent on the photoexcited carrier density [49], as well as on substrate-induced charges or strain. By keeping the excitation power density at a constant low level of approximately $5 \frac{\mu \mathrm{W}}{\mu \mathrm{m}^{2}}$ for all our photoluminescence measurements, we can rule out the effect of photoexcited carrier densities as the origin of the observed phenomena. In the following, we want to discuss substrate-induced charges and strain as possible origins for our observations, namely the redshifts in the optical band gaps and the reductions in the excitonic binding energies.

In our experiments, we expect the discrepancy in the thermal expansion coefficients of the substrates and the TMD-MLs as the main source of strain when cooling down our samples to cryogenic temperatures. As outlined in the Supplemental Material [30], most of the substrate materials show lower thermal expansion coefficients than the TMD materials in this work. Accordingly, tensile strain can be expected, e.g., for a TMD-ML on h-BN, when the sample is cooled down. For tensile strain redshifts in the optical band gap of a TMD-ML are expected [41]. However, although, we cannot fully rule out that strain contributed to the observed effect of substrate on exciton and trion emission wavelengths, it cannot be the main factor. The following arguments strengthen the conclusion that dielectric effects are the driving force here: (i) the van der Waals bonding between the TMD-ML and the substrate are expected to minimize a strain transfer to the ML; (ii) the redshift for TMD-MLs encapsulated with $\mathrm{h}-\mathrm{BN}$ is approximately twice as big as for TMD-MLs on h-BN, which cannot be explained by strain; and (iii) excitonic binding energies are only very weakly affected by strain [50], however, in our experiments they change together with the optical band gaps. In order to fully decouple dielectric effects from strain effects our future plans include the application of further experimental methods such as Raman spectroscopy and $x$-ray diffraction spectroscopy.

Screening of Coulomb interactions at high carrier densities in TMD-MLs can lead to shifts in the exciton energies [49]. Such charges can be unintentionally introduced, for example, via substrate doping [51]. In order to evaluate the possible role of doping we performed gate dependent photoluminescence experiments by contacting the device shown in Fig. 6 with graphene as a contact. The results of the gate dependent photoluminescence experiments are shown in the Supplemental Material, Fig. 2 [30]. It is apparent that, although the emission energies of both the neutral exciton and the trion are undergoing small changes if the gate voltage is changed, they are much smaller than the effect of the dielectric environment. In addition, different binding energies for negatively and positively charged trions can be seen, supporting the suggestion that the measured trion binding energy for a $\mathrm{MoSe}_{2} \mathrm{ML}$ on CYTOP can be explained by a positive polarity of the trion in this structure.

\section{CONCLUSIONS}

We have shown that $\mathrm{h}-\mathrm{BN}$ is a suitable cladding material to tune the optical band gap of TMD-MLs, whereas oxide substrates, irrespective of their relative permittivities, do not lead to a significant change in the optical band gaps of TMD-MLs.

A reduction of the optical band gaps of $\mathrm{MoSe}_{2}$ and $\mathrm{WSe}_{2}$ MLs by $37 \mathrm{meV}$ was measured between the uncovered ML placed on a CYTOP substrate and a ML encapsulated in $\mathrm{h}-\mathrm{BN}$. In addition, relative changes in the energy splittings of excited excitonic states in $\mathrm{WSe}_{2}$ MLs of approximately $30 \%$ were measured. From these results, a maximum reduction of $115 \mathrm{meV}$ and a minimum reduction of $150 \mathrm{meV}$ are expected for the exciton binding energy and the electronic band gap, respectively, of a $\mathrm{WSe}_{2} \mathrm{ML}$ when a CYTOP substrate is replaced with a h-BN encapsulation.

The energies of optical transitions obtained in this work have been probed on the time scale of the exciton lifetime. It would be interesting to compare our results for the electronic band gap with scanning tunneling microscopy experiments, which probe the electronic band gap in the static limit.

We have shown that formation of optical lateral heterostructure purely by local variation of screening in three dimensions will require a control of the substrate flatness as well as of the environment induced charge state.

In the heterojunction studied in this work (see Fig. 6), the change from one region to another region was abrupt on the scale of the probe beam. Therefore, techniques with a better spatial resolution need to be developed in order to study dielectric effects on smaller length scales.

Our findings pave the way towards the creation of controlled lateral heterostructures, which are required as building blocks for next generation electronic and optoelectronic devices.

\section{ACKNOWLEDGMENTS}

We would like to thank Tony Heinz, Alexey Chernikov, and Archana Raja for fruitful discussions and helpful advices.
[1] K. S. Novoselov, A. K. Geim, S. V. Morozov, D. Jiang, Y. Zhang, S. V. Dubonos, I. V. Grigorieva, and A. A. Firsov, Electric field effect in atomically thin carbon films, Science 306, 666 (2004).
[2] L. Li, Y. Yu, G. J. Ye, Q. Ge, X. Ou, H. Wu, D. Feng, X. H. Chen, and Y. Zhang, Black phosphorus field-effect transistors, Nat. Nanotechnol. 9, 372 (2014). 
[3] P. Vogt, P. D. Padova, C. Quaresima, J. Avila, E. Frantzeskakis, M. C. Asensio, A. Resta, B. Ealet, and G. L. Lay, Silicene: Compelling Experimental Evidence for Graphenelike TwoDimensional Silicon, Phys. Rev. Lett. 108, 155501 (2012).

[4] M. E. Dávila, L. Xian, S. Cahangirov, A. Rubio, and G. L. Lay, Germanene: A novel two-dimensional germanium allotrope akin to graphene and silicene, New J. Phys. 16, 095002 (2014).

[5] K. F. Mak, C. Lee, J. Hone, J. Shan, and T. F. Heinz, Atomically Thin $\mathrm{Mos}_{2}$ : A New Direct-Gap Semiconductor, Phys. Rev. Lett. 105, 136805 (2010).

[6] F. A. Rasmussen and K. S. Thygesen, Computational 2d materials database: Electronic structure of transition-metal dichalcogenides and oxides, J. Phys. Chem. C 119, 13169 (2015).

[7] M. M. Ugeda, A. J. Bradley, S.-F. Shi, F. H. da Jornada, Y. Zhang, D. Y. Qiu, W. Ruan, S.-K. Mo, Z. Hussain, Z.-X. Shen, F. Wang, S. G. Louie, and M. F. Crommie, Giant bandgap renormalization and excitonic effects in a monolayer transition metal dichalcogenide semiconductor, Nat. Mater. 13, 1091 (2014).

[8] T. Cao, G. Wang, W. Han, H. Ye, C. Zhu, J. Shi, Q. Niu, P. Tan, E. Wang, B. Liu, and J. Feng, Valley-selective circular dichroism of monolayer molybdenum disulphide, Nat. Commun. 3, 887 (2012).

[9] D. Xiao, G.-B. Liu, W. Feng, X. Xu, and W. Yao, Coupled Spin and Valley Physics in Monolayers of $\mathrm{MoS}_{2}$ and Other Group-VI Dichalcogenides, Phys. Rev. Lett. 108, 196802 (2012).

[10] B. Radisavljevic, A. Radenovic, J. Brivio, V. Giacometti, and A. Kis, Single-layer $\mathrm{MoS}_{2}$ transistors, Nat. Nanotechnol. 6, 147 (2011).

[11] J. S. Ross, P. Klement, A. M. Jones, N. J. Ghimire, J. Yan, D. G. Mandrus, T. Taniguchi, K. Watanabe, K. Kitamura, W. Yao, D. H. Cobden, and X. Xu, Electrically tunable excitonic light-emitting diodes based on monolayer $\mathrm{WSe}_{2} \mathrm{p}-\mathrm{n}$ junctions, Nat. Nanotechnol. 9, 268 (2014).

[12] O. Lopez-Sanchez, D. Lembke, M. Kayci, A. Radenovic, and A. Kis, Ultrasensitive photodetectors based on monolayer $\mathrm{MoS}_{2}$, Nat. Nanotechnol. 8, 497 (2013).

[13] C. Huang, S. Wu, A. M. Sanchez, J. J. P. Peters, R. Beanland, J. S. Ross, P. Rivera, W. Yao, D. H. Cobden, and X. Xu, Lateral heterojunctions within monolayer $\mathrm{MoSe}_{2}-\mathrm{WSe}_{2}$ semiconductors, Nat. Mater. 13, 1096 (2014).

[14] M. Mahjouri-Samani, M.-W. Lin, K. Wang, A. R. Lupini, J. Lee, L. Basile, A. Boulesbaa, C. M. Rouleau, A. A. Puretzky, I. N. Ivanov, K. Xiao, M. Yoon, and D. B. Geohegan, Patterned arrays of lateral heterojunctions within monolayer two-dimensional semiconductors, Nat. Commun. 6, 7749 (2015).

[15] M. Rösner, C. Steinke, M. Lorke, C. Gies, F. Jahnke, and T. O. Wehling, Two-dimensional heterojunctions from nonlocal manipulations of the interactions, Nano Lett. 16, 2322 (2016).

[16] J. Ryou, Y.-S. Kim, S. KC, and K. Cho, Monolayer MoS bandgap modulation by dielectric environments and tunable bandgap transistors, Sci. Rep. 6, 29184 (2016).

[17] J. S. Ross, S. Wu, H. Yu, N. J. Ghimire, A. M. Jones, G. Aivazian, J. Yan, D. G. Mandrus, D. Xiao, W. Yao, and X. Xu, Electrical control of neutral and charged excitons in a monolayer semiconductor, Nat. Commun. 4, 1474 (2013).

[18] K. F. Mak, K. He, J. Shan, and T. F. Heinz, Control of valley polarization in monolayer $\mathrm{MoS}_{2}$ by optical helicity, Nat. Nanotechnol. 7, 494 (2012).
[19] G. Wang, L. Bouet, D. Lagarde, M. Vidal, A. Balocchi, T. Amand, X. Marie, and B. Urbaszek, Valley dynamics probed through charged and neutral exciton emission in monolayer $\mathrm{WSe}_{2}$, Phys. Rev. B 90, 075413 (2014).

[20] G. Plechinger, P. Nagler, J. Kraus, N. Paradiso, C. Strunk, C. Schüller, and T. Korn, Identification of excitons, trions and biexcitons in single-layer $\mathrm{WS}_{2}$, Phys. Status Solidi (RRL) 9 , 457 (2015).

[21] A. Chernikov, T. C. Berkelbach, H. M. Hill, A. Rigosi, Y. Li, O. B. Aslan, D. R. Reichman, M. S. Hybertsen, and T. F. Heinz, Exciton Binding Energy and Nonhydrogenic Rydberg Series in Monolayer $\mathrm{WS}_{2}$, Phys. Rev. Lett. 113, 076802 (2014).

[22] K. He, N. Kumar, L. Zhao, Z. Wang, K. F. Mak, H. Zhao, and J. Shan, Tightly Bound Excitons in Monolayer WSe $\mathrm{W}_{2}$, Phys. Rev. Lett. 113, 026803 (2014).

[23] H. M. Hill, A. F. Rigosi, C. Roquelet, A. Chernikov, T. C. Berkelbach, D. R. Reichman, M. S. Hybertsen, L. E. Brus, and T. F. Heinz, Observation of excitonic Rydberg states in monolayer $\mathrm{MoS}_{2}$ and $\mathrm{WS}_{2}$ by photoluminescence excitation spectroscopy, Nano Lett. 15, 2992 (2015).

[24] S. Latini, T. Olsen, and K. S. Thygesen, Excitons in van der Waals heterostructures: The important role of dielectric screening, Phys. Rev. B 92, 245123 (2015).

[25] I. Kylänpää and H.-P. Komsa, Binding energies of exciton complexes in transition metal dichalcogenide monolayers and effect of dielectric environment, Phys. Rev. B 92, 205418 (2015).

[26] Y. Lin, X. Ling, L. Yu, S. Huang, A. L. Hsu, Y.-H. Lee, J. Kong, M. S. Dresselhaus, and T. Palacios, Dielectric screening of excitons and trions in single-layer $\mathrm{MoS}_{2}$, Nano Lett. 14, 5569 (2014).

[27] A. V. Stier, N. P. Wilson, G. Clark, X. Xu, and S. A. Crooker, Probing the influence of dielectric environment on excitons in monolayer $\mathrm{WSe}_{2}$ : Insight from high magnetic fields, Nano Lett. 16, 7054 (2016).

[28] A. Raja, A. Chaves, J. Yu, G. Arefe, H. M. Hill, A. F. Rigosi, T. C. Berkelbach, P. Nagler, C. Schüller, T. Korn, C. Nuckolls, J. Hone, L. E. Brus, T. F. Heinz, D. R. Reichman, and A. Chernikov, Coulomb engineering of the bandgap and excitons in two-dimensional materials, Nat. Commun. 8, 15251 (2017).

[29] S. Lippert, L. M. Schneider, D. Renaud, K. N. Kang, O. Ajayi, J. Kuhnert, M.-U. Halbich, O. M. Abdulmunem, X. Lin, and $\mathrm{K}$. Hassoon, Influence of the substrate material on the optical properties of tungsten diselenide monolayers, 2D Mater. 4, 025045 (2017).

[30] See Supplemental Material at http://link.aps.org/supplemental/ 10.1103/PhysRevMaterials.1.054001 for further discussions on sample preparation, dielectric functions, strain and charging effects.

[31] F. Cadiz, E. Courtade, C. Robert, G. Wang, Y. Shen, H. Cai, T. Taniguchi, K. Watanabe, H. Carrere, D. Lagarde, M. Manca, T. Amand, P. Renucci, S. Tongay, X. Marie, and B. Urbaszek, Excitonic Linewidth Approaching the Homogeneous Limit in $\mathrm{MoS}_{2}$-Based van der Waals Heterostructures, Phys. Rev. X 7, 021026 (2017).

[32] O. A Ajayi, J. V. Ardelean, G. D. Shepard, J. Wang, A. Antony, T. Taniguchi, K. Watanabe, T. F. Heinz, S. Strauf, and X.-Y. $\mathrm{Zhu}$, Approaching the intrinsic photoluminescence linewidth in transition metal dichalcogenide monolayers, 2D Mater. 4, 031011 (2017). 
[33] C. Jin, J. Kim, J. Suh, Z. Shi, B. Chen, X. Fan, M. Kam, K. Watanabe, T. Taniguchi, S. Tongay, A. Zettl, J. Wu, and F. Wang, Interlayer electron-phonon coupling in $\mathrm{WSe}_{2} / \mathrm{h}-\mathrm{BN}$ heterostructures, Nat. Phys. 13, 127 (2017).

[34] C. M. Chow, H. Yu, A. M. Jones, J. Yan, D. G. Mandrus, T. Taniguchi, K. Watanabe, W. Yao, and X. Xu, Unusual excitonphonon interactions at van der Waals engineered interfaces, Nano Lett. 17, 1194 (2017).

[35] A. M. Jones, H. Yu, N. J. Ghimire, S. Wu, G. Aivazian, J. S. Ross, B. Zhao, J. Yan, D. G. Mandrus, D. Xiao, W. Yao, and X. Xu, Optical generation of excitonic valley coherence in monolayer $\mathrm{WSe}_{2}$, Nat. Nanotechnol. 8, 634 (2013).

[36] K. Kobayashl, Optical spectra and electronic structure of ice, J. Phys. Chem. 87, 4317 (1983).

[37] S. J. Haigh, A. Gholinia, R. Jalil, S. Romani, L. Britnell, D. C. Elias, K. S. Novoselov, L. A. Ponomarenko, A. K. Geim, and R. Gorbachev, Cross-sectional imaging of individual layers and buried interfaces of graphene-based heterostructures and superlattices, Nat. Mater. 11, 764 (2012).

[38] H. Shi, H. Pan, Y.-W. Zhang, and B. I. Yakobson, Quasiparticle band structures and optical properties of strained monolayer $\mathrm{MoS}_{2}$ and $\mathrm{WS}_{2}$, Phys. Rev. B 87, 155304 (2013).

[39] P. K. Chow, E. Singh, B. C. Viana, J. Gao, J. Luo, J. Li, Z. Lin, A. L. Elías, Y. Shi, Z. Wang, M. Terrones, and N. Koratkar, Wetting of mono and few-layered $\mathrm{WS}_{2}$ and $\mathrm{MoS}_{2}$ films supported on $\mathrm{Si} / \mathrm{SiO}_{2}$ substrates, ACS Nano 9, 3023 (2015).

[40] P. Johari and V. B. Shenoy, Tuning the electronic properties of semiconducting transition metal dichalcogenides by applying mechanical strains, ACS Nano 6, 5449 (2012).

[41] K. He, C. Poole, K. F. Mak, and J. Shan, Experimental demonstration of continuous electronic structure tuning via strain in atomically thin $\mathrm{MoS}_{2}$, Nano Lett. 13, 2931 (2013).

[42] D. Lloyd, X. Liu, J. W. Christopher, L. Cantley, A. Wadehra, B. L. Kim, B. B. Goldberg, A. K. Swan, and J. S. Bunch, Band gap engineering with ultralarge biaxial strains in suspended monolayer $\mathrm{MoS}_{2}$, Nano Lett. 16, 5836 (2016).

[43] Multilayer thin film optics calculator, http://sjbyrnes.com/ (2012).

[44] G. Wang, I. C. Gerber, L. Bouet, D. Lagarde, A. Balocchi, M. Vidal, T. Amand, X. Marie, and B. Urbaszek, Exciton states in monolayer $\mathrm{MoSe}_{2}$ : Impact on interband transitions, 2D Mater. 2, 045005 (2015).

[45] C. Steinke, D. Mourad, M. Rosner, M. Lorke, C. Gies, F. Jahnke, G. Czycholl, and T. O. Wehling, Noninvasive control of excitons in two-dimensional materials, Phys. Rev. B 96, 045431 (2017).

[46] G. Wang, X. Marie, I. Gerber, T. Amand, D. Lagarde, L. Bouet, M. Vidal, A. Balocchi, and B. Urbaszek, Giant Enhancement of the Optical Second-Harmonic Emission of $\mathrm{WSe}_{2}$ Monolayers by Laser Excitation at Exciton Resonances, Phys. Rev. Lett. 114, 097403 (2015).

[47] K. Andersen, S. Latini, and K. S. Thygesen, Dielectric genome of van der Waals heterostructures, Nano Lett. 15, 4616 (2015).

[48] Y. Zhang, T.-R. Chang, B. Zhou, Y.-T. Cui, H. Yan, Z. Liu, F. Schmitt, J. Lee, R. Moore, Y. Chen, H. Lin, H.-T. Jeng, S.-K. Mo, Z. Hussain, A. Bansil, and Z.-X. Shen, Direct observation of the transition from indirect to direct bandgap in atomically thin epitaxial $\mathrm{MoSe}_{2}$, Nat. Nanotechnol. 9, 111 (2014).

[49] A. Steinhoff, M. Rösner, F. Jahnke, and T. O. W. C. Gies, Influence of excited carriers on the optical and electronic properties of $\mathrm{MoS}_{2}$, Nano Lett. 14, 3743 (2014).

[50] M. Feierabend, A. Morlet, G. Berghäuser, and E. Malic, Impact of strain on the optical fingerprint of monolayer transition metal dichalcogenides, Phys. Rev. B 96, 045425 (2017).

[51] D. Sercombe, S. Schwarz, O. D. Pozo-Zamudio, F. Liu, B. J. Robinson, E. A. Chekhovich, I. I. Tartakovskii, O. Kolosov, and A. I. Tartakovskii, Optical investigation of the natural electron doping in thin $\mathrm{MoS}_{2}$ films deposited on dielectric substrates, Sci. Rep. 3, 3489 (2013). 